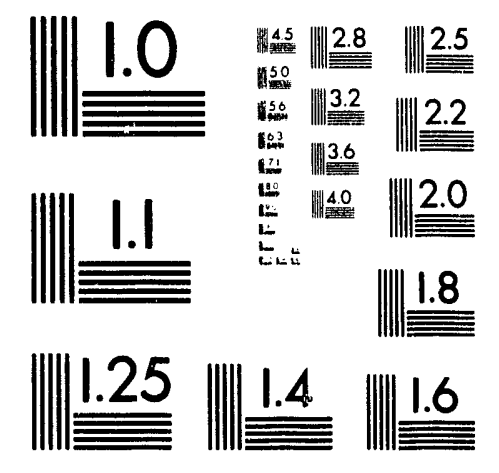



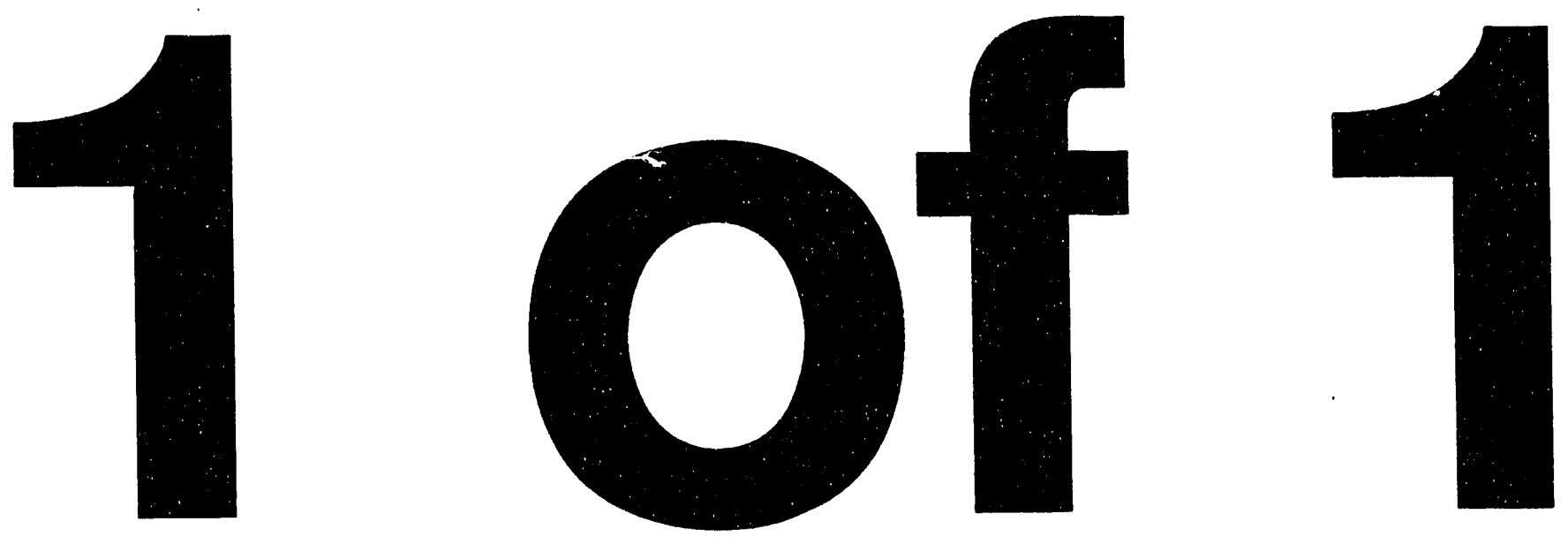


\title{
ALTERNATING-PHASE FOCUSING WITH AMPLITUDE MODULATION
}

\author{
L. Sagalovsky, J. R. Delayen \\ Engineering Physics Division, Argonne National Laboratory \\ Argonne, Illinois 60439 USA \\ $\cos 3$
}

\section{Abstract}

We have previously developed a model of alternating-phase focusing (APF) applicable to ion linacs comprised of short independently controlled cavities. The main beam dynamical aspects of APF are adequately described by four parameters: equilibrium synchronous phase, phase modulation amplitude, length of APF period, and incremental energy gain. In this paper we report on an extension of the analysis to include simultaneous modulation of the accelerating field amplitude. Two additional parameters are included: relative phase between the amplitude and phase modulation and magnitude of the amplitude modulation. The effects of amplitude modulation on the stable regions and longitudinal acceptance are discussed.

\section{INTRODUCTION}

In our previous paper [.., , we developed an analytical model suitable for studyir o APF beam dynamics in low-energy superconducting linacs. We derived equations of motion for the electric field described by a cylindrically symmetric travelling wave with a continuous phase modulation and obtained approximate analytical solutions for both the regic ns of linear stability and the nonlinear longitudinal acceptance. In this paper, we generalize the model to include a simultaneous modulation of the acceleration amplitude. Early works in the field [2] suggested that the amplitude modulation may increase the effectiveness of APF. We show how the effect can be quantified in the context of our model.

\footnotetext{
-Work supported by the U. S. Army Strategic Defense Command
} under the auspices of the U.S. Department of Energy.

\section{APF BEAM DYNamics}

\section{A. Equations of Motion}

We choose a sinusoidal modulation for both the phase and the amplitude of the electric field:

$$
\begin{aligned}
E_{z}= & E_{0}\left[1+\epsilon \sin \left(\int_{0}^{z} \frac{2 \pi}{\Lambda} d z^{\prime}+\delta\right)\right] \\
& \times \cos \left[\omega t-\int_{0}^{z} k\left(z^{\prime}\right) d z^{\prime}+\phi_{0}+\phi_{1} \sin \left(\int_{0}^{z} \frac{2 \pi}{\Lambda} d z^{\prime}\right)\right],
\end{aligned}
$$

where $\omega$ is the angular velocity and $k$ is the wave number of the rf field, $\phi_{0}$ is the equilibrium phase, $\Lambda$ and $\phi_{1}$ are the APF period and phase modulation amplitude respectively, and $\epsilon$ and $\delta$ are the amplitude modulation strength and relative phase respectively. For the central reference trajectory $z_{c}$, we take

$$
\omega t-\int_{0}^{z_{c}} k\left(z^{\prime}\right) d z^{\prime}=0 .
$$

We will use the following dimensionless variables to analyze APF throughout the paper,

$$
\Delta \phi \equiv-k \Delta z, \tau \equiv \int_{0}^{z_{\mathrm{c}}} \frac{d z}{\Lambda}, \nu \equiv \frac{\Lambda}{\beta \lambda},
$$

and

$$
\eta \equiv \frac{q E_{0} \beta \lambda}{m \beta^{2} c^{2} / 2}, \quad\langle\eta\rangle \equiv \frac{q\langle E\rangle \beta \lambda}{m \beta^{2} c^{2} / 2},
$$

where $\langle E\rangle$ is the accelerating gradient obtained by averaging $E_{z}$ given by eq. 2 over one APF period,

$$
\langle E\rangle=E_{0}\left[J_{0}\left(\phi_{1}\right) \cos \phi_{0}-\epsilon J_{1}\left(\phi_{1}\right) \sin \phi_{0} \cos \delta\right] .
$$

Following derivations in ref. [1], we get the equation of motion for the longitudinal coordinate $\Delta \phi$,

$$
\begin{aligned}
\frac{d^{2} \Delta \phi}{d \tau^{2}}= & -\pi \eta \nu^{2}[(c(\Delta \phi)-c(0)) \\
& +\epsilon(c(\Delta \phi) s(\Delta \phi)-c(0) s(0))],
\end{aligned}
$$

where

$$
\begin{aligned}
& c(\Delta \phi)=\cos \left[\phi_{0}+\Delta \phi+\phi_{1} \sin \left(2 \pi \tau-\frac{\Delta \phi}{\nu}\right)\right] \\
& s(\Delta \phi)=\sin \left[\sin \left(2 \pi \tau-\frac{\Delta \phi}{\nu}+\delta\right)\right]
\end{aligned}
$$


In the transverse direction, we confine our attention to the linear motion only. The linear equation in the radial coordinate $r$ will be given in the next section. It is obtained from using Maxwell's equations which to the first order in $r$ relate the transverse and the longitudinal field component, $E_{r}(r, z ; t)=-r / 2 \quad \partial E_{z} / \partial z$.

\section{B. Regions of Linear Stability}

Linearized equations of motion can be written as follows,

$$
\begin{aligned}
\frac{d^{2} \Delta \phi}{d \tau^{2}} & =\pi \eta \nu\left[\left(\nu-\phi_{1} \cos 2 \pi \tau\right) \sin \left(\phi_{0}+\phi_{1} \sin 2 \pi \tau\right)\right] \Delta \phi \\
\frac{d^{2} r}{d \tau^{2}} & =-\frac{\pi}{2} \eta \nu\left[\left(\nu-\phi_{1} \cos 2 \pi \tau\right) \sin \left(\phi_{0}+\phi_{1} \sin 2 \pi \tau\right)\right] r .
\end{aligned}
$$

Keeping only the $n=1$ term, we obtain the well-known Mathieu equations

$$
\begin{gathered}
\frac{d^{2} \Delta \phi}{d \tau^{2}}-2\left[B+C_{1} \sin \left(2 \pi \tau+\theta_{1}\right)\right] \Delta \phi=0 \\
\frac{d^{2} r}{d \tau^{2}}+\left[B+C_{1} \sin \left(2 \pi \tau+\theta_{1}\right)\right] r=0
\end{gathered}
$$

for which we can compute stable region boundaries. The coefficients $B$ and $C_{1}$ can be computed explicitly in terms of the APF parameters,

$$
\begin{gathered}
B=\frac{\pi}{2} \eta \nu^{2}\left[J_{0}\left(\phi_{1}\right) \sin \phi_{0}+\epsilon J_{1}\left(\phi_{1}\right) \cos \phi_{0} \cos \delta\right] \\
C_{1}=-\pi \eta \nu\left|J_{1}\left(\phi_{1}\right)\right| \cos \phi_{0} \sqrt{\nu^{2}+\tan ^{2} \phi_{0}} \xi(\epsilon),
\end{gathered}
$$

where

$$
\xi(\epsilon)=1-\frac{\epsilon}{\phi_{1}} \frac{\left(1-\nu^{2}\right) \sin 2 \phi_{0} \cos \delta+2 \nu \sin \delta}{2\left(\sin ^{2}\right.} \frac{\left.\phi_{0}+\nu^{2} \cos ^{2} \phi_{0}\right)}{O}+O\left(\epsilon^{2}\right) .
$$

Fig. 1 (a) shows stability boundaries in the $\phi_{1}-\nu$ plane for $\phi_{0}=5^{\circ},\langle\eta\rangle=0.25$ (parameter $\langle\eta\rangle$ represents the relative energy change over $\beta \lambda$ ) with no amplitude modulation. Figs. 1 (b), (c), (d) show the effect of modulating the field amplitude with $\epsilon=0.1$ and the relative phase $\delta$ of $0^{\circ}, 90^{\circ}$, and $180^{\circ}$. The shift of the stable region to lower values of $\phi_{1}$ is desirable because it allows to use smaller electric field strength $E_{0}$ to achieve the same accelerating gradient in the APF cell (cf. eq. 5).

\section{Calculation of Longitudinal Acceptance}

Eq. 6 can be expanded in a Fourier series and written in the most general form as follows,

$$
\frac{d^{2} \Delta \phi}{d \tau^{2}}=-\frac{\partial U_{0}}{\partial \Delta \phi}+\sum_{n=1}^{\infty}\left[u_{n} \sin (2 \pi n \tau)+v_{n} \cos (2 \pi n \tau)\right]
$$

Solution to eq. 16 can be expressed as a sum of two components,

$$
\Delta \phi(\tau)=\langle\Delta \phi(\tau)\rangle+\delta(\Delta \phi(\tau))
$$
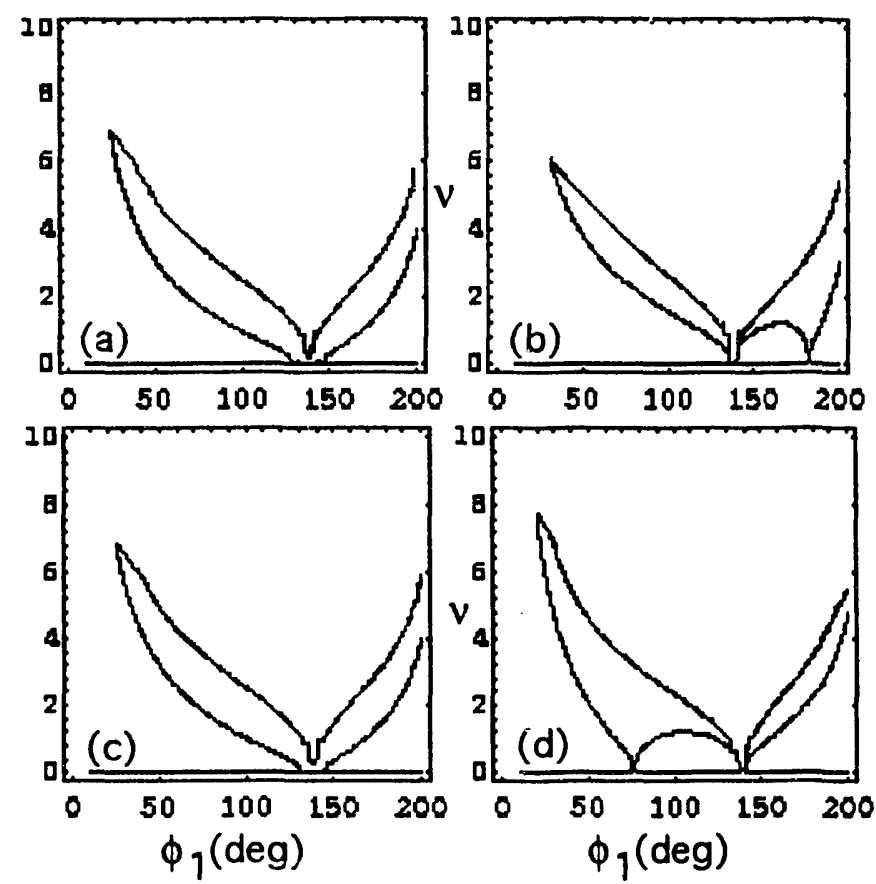

Figure 1: Stability boundaries for trajectories not exceeding $90^{\circ}$ in either transverse or longitudinal phase advance with $\phi_{0}=5^{\circ}$ and $\langle\eta\rangle=0.25$ for (a) $\epsilon=0$, (b) $\epsilon=0.1$, $\delta=0$, (c) $\epsilon=0.1, \delta=\frac{\pi}{2}$, (d) $\epsilon=0.1, \delta=\pi$.

where $\langle\Delta \phi(\tau)\rangle$ represents the average motion and $\delta(\Delta \phi(\tau))$ represents the fluctuations caused by the rapidly oscillating force (from here on we will drop the \langle\rangle when denoting the slowly varying solution). Following the prescription given in ref. [3] and applied to the problem of longitudinal acceptance in ref. [4], we can obtain the timeaveraged equation of motion as

$$
\frac{d^{2} \Delta \phi}{d \tau^{2}}=-\frac{d U_{\mathrm{eff}}}{d \Delta \phi}
$$

where

$$
U_{\text {eff }}=U_{0}+\frac{1}{16 \pi^{2}} \sum_{n=1}^{\infty} \frac{u_{n}^{2}+v_{n}^{2}}{n^{2}}
$$

The potential function $U_{0}$ and the Fourier coefficients $u_{n}$, $v_{n}$ can be written as follows,

$$
U_{0}=U_{0}^{0}+\epsilon U_{0}^{1}, \quad u_{n}=u_{n}^{0}+\epsilon u_{n}^{1}, \quad v_{n}=v_{n}^{0}+\epsilon v_{n}^{1},
$$

where $U_{0}^{0}, u_{n}^{0}, v_{n}^{0}$ were given explicitly in ref. [1] and

$$
U_{0}^{1}=\pi \eta \nu^{2} J_{1}\left(\phi_{1}\right) \cos \delta\left(c_{1}+s_{0} \Delta \phi-c_{0}\right),
$$

$$
u_{n}^{1}=-\pi \eta \nu^{2}\left\{\begin{array}{ll}
\frac{2 n}{\phi_{1}} J_{n}\left(\phi_{1}\right)\left(c_{1} c_{-1}^{\delta}-c_{0} c_{0}^{\delta}\right) & \text { if } n \text { odd } \\
-J_{n-1}\left(\phi_{1}\right)\left(s_{1} s_{-1}^{\delta}-s_{0} s_{0}^{\delta}\right) & \\
-J_{n+1}\left(\phi_{1}\right)\left(s_{1} s_{+1}^{\delta}-s_{0} s_{0}^{\delta}\right) & \text { if } n \text { even }
\end{array}\right. \text {, }
$$$$
v_{n}^{1}=-\pi \eta \nu^{2} \begin{cases}\frac{2 n}{\phi_{1}} J_{n}\left(\phi_{1}\right)\left(c_{1} s_{-1}^{\delta}-c_{0} s_{0}^{\delta}\right) & \text { if } n \text { odd } \\ J_{n-1}\left(\phi_{1}\right)\left(s_{1} c_{-1}^{\delta}-s_{0} c_{0}^{\delta}\right) & \\ -J_{n+1}\left(\phi_{1}\right)\left(s_{1} c_{+1}^{\delta}-s_{0} c_{0}^{\delta}\right) & \text { if } n \text { even }\end{cases}
$$ 


$$
\begin{gathered}
r=\cos \phi_{0}, \quad c_{1}=\cos \left(\phi_{0}+\Delta \phi\right), \\
s_{0}=\sin \phi_{0}, \quad s_{1}=\sin \left(\phi_{0}+\Delta \phi\right), \\
c_{0}^{\delta}=\cos \delta, \quad c_{ \pm 1}^{\delta}=\cos \left(\delta \pm \frac{n}{\nu} \Delta \phi\right), \\
s_{0}^{\delta}=\sin \delta, \quad s_{ \pm 1}^{\delta}=\sin \left(\delta \pm \frac{n}{\nu} \Delta \phi\right) .
\end{gathered}
$$

Given the effective potential, we can calculate the width of the separatrix in the $\left(\Delta \phi, \frac{\Delta W}{W}\right)$ space and the longitudinal acceptance. The equations are found in ref. [1]. Below we examine the solution accurate to $O\left(\Delta \phi^{2}\right)$.

1) Second-Order Solution: The effective potential $U_{\text {eff }}$ can be expanded to $O\left(\Delta \phi^{3}\right)$ to yield

$$
U_{\mathrm{eff}}(\Delta \phi)=\frac{a}{2} \Delta \phi^{2}-\frac{b}{3} \Delta \phi^{3}+\cdots,
$$

where $a$ is the square of the linear phase advance $\sigma_{L}$,

$$
a=\sigma_{L}^{2} \simeq 2 B+\frac{1}{2 \pi^{2}} C_{1}^{2}
$$

and $b$ is given by

$$
\begin{aligned}
b \simeq & \frac{\pi}{2} \eta \nu^{2}\left[J_{0}\left(\phi_{1}\right) \cos \phi_{0}-\epsilon \cos \delta J_{1}\left(\phi_{1}\right) \sin \phi_{0}\right] \\
& +\frac{3}{8} \eta^{2} \nu^{2}\left(1-\nu^{2}\right) J_{1}^{2}\left(\phi_{1}\right) \sin 2 \phi_{0} \\
& \times\left[1+2 \epsilon \frac{\cot 2 \phi_{0}}{\phi_{1}} \cos \delta+O\left(\epsilon^{2}\right)\right] .
\end{aligned}
$$

The width of the separatrix $\Psi$ and the acceptance $\alpha_{L}$ are calculated to be

$$
\Psi=\frac{3}{2} \frac{a}{b}, \quad \alpha_{L}=\frac{6}{5 \pi \nu} \frac{a^{5 / 2}}{b^{2}} .
$$

We have kept only the $n=1$ terms in eqs. 25,27 .

Figs. 2(a) and 2(b) show the effect of varying the relative phase $\delta$ on the separatrix width and the acceptance respectively for different values of $\epsilon$. The calculations were performed for $\langle\eta\rangle=0.25, \phi_{0}=5^{\circ}, \phi_{1}=60^{\circ}$, and $\nu=4$. For $\epsilon=0.1$ the separatrix can be widened by $20 \%$ with a corresponding $67 \%$ increase in the acceptance; for $\epsilon=0.2$ the respective numbers are $33 \%$ and $147 \%$.

\section{Conclusions}

The enhanced model of the traveling wave with continuous phase and amplitude modulation presented in this paper gives quantitative predictions to the problem of longitudinal stability in APF linacs. The model describes the physics of APF with six parameters and yields analytic solutions for the stable regions and the longitudinal acceptance.

Effects of modulating accelerating field amplitude can be computed explicitly keeping only quadratic terms in the expression for the time-averaged APF force. Relative
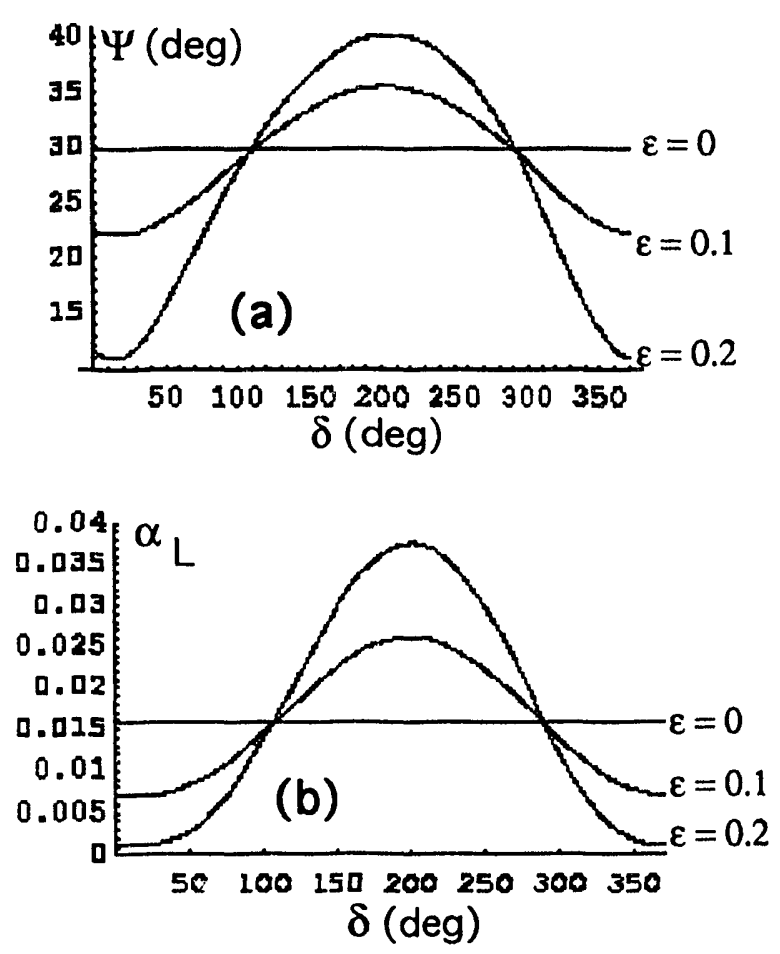

Figure 2: Plots of (a) the separatrix width $\Psi$ and (b) the longitudinal acceptance $\alpha_{L}$ for $\langle\eta\rangle=0.25, \phi_{0}=5^{\circ}, \phi_{1}=$ $60^{\circ}, \nu=4$ as a function of the relative phase $\delta$ for various values of the strength parameter $\epsilon$.

phase between the amplitude and phase modulations determines the degree of the acceptance enhancement (or reduction). Many different scenarios can be investigated in the context of the model.

Future work will focus on investigations of practical limits of APF in linacs with independent superconducting cavities and space-charge current limits.

\section{REFERENCES}

[1] L. Sagalovsky, J. R. Delayen, Proc. 1992 Linear Accelerator Conf., AECL - 10728, 763 (1992).

[2] V. V. Kushin, V. M. Mokhov, Atomnaya Energiya 35 (3) (1973).

[3] P. L. Kapitsa, Zh. Eksper. Teor. Fiz. 21, 588 (1951).

[4] H. Okamoto, Nucl. Instrum. Methods A284, 233-247 (1989). 

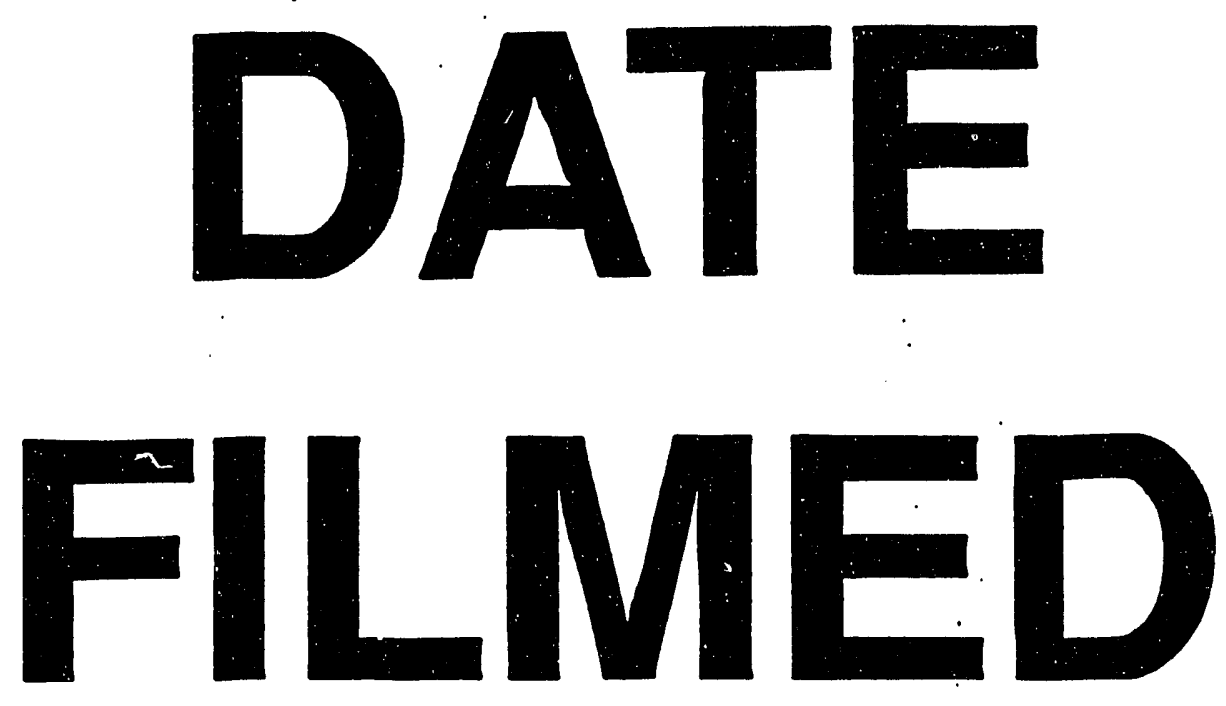

$10 / / 4 / 93$
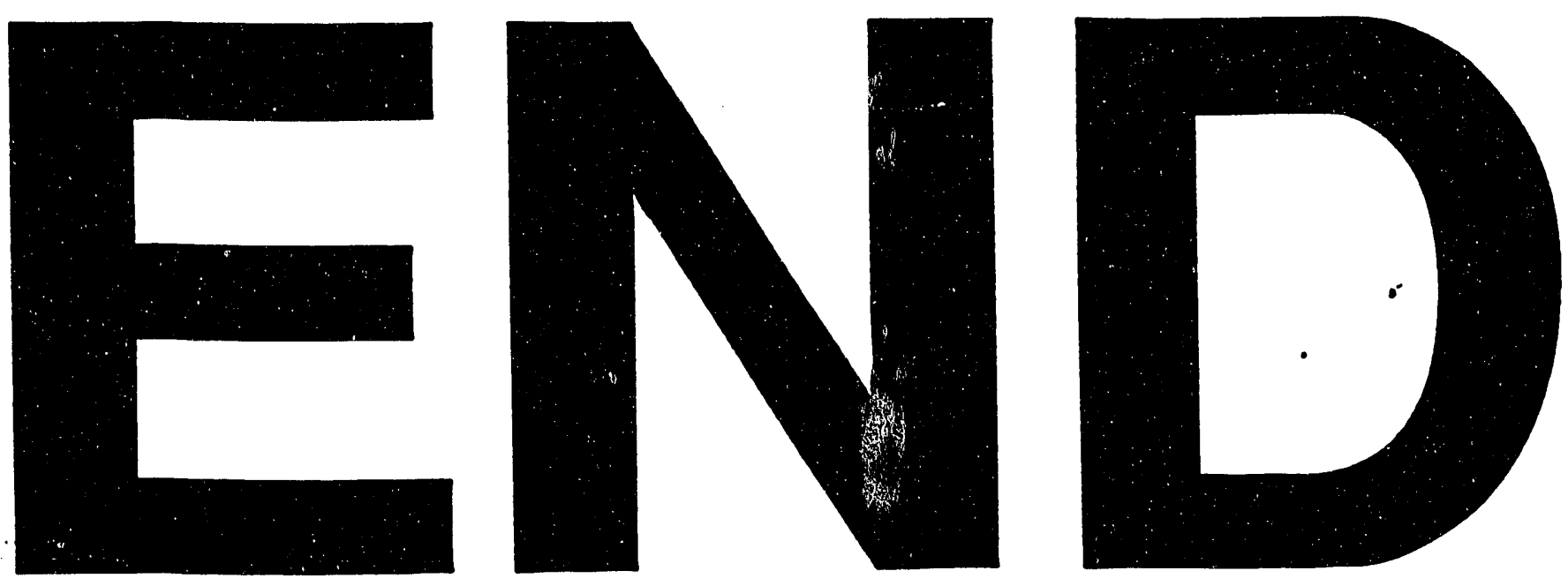
